\title{
PRIMARY RESEARCH \\ Over-speeding warning system using wireless communications for road signs and vehicles
}

\author{
Samuel N. Cubero ${ }^{1 *}$, Simon McLernon ${ }^{2}$, Aaron Sharpe ${ }^{3}$
}

1, General Studies, College of Arts and Science, The Petroleum Institute, Abu Dhabi, United Arab Emirates (UAE)

${ }^{2,3}$ Department of Mechanical Engineering, Curtin University of Technology, Perth, WA, Australia

\author{
Index Terms \\ Over-Speeding \\ Vehicle \\ Wireless \\ Communications \\ Microcontroller \\ Automobile \\ Road Safety
}

Received: 11 June 2016

Accepted: 12 July 2016

Published: 27 October 2016

\begin{abstract}
The problem of exceeding the posted speed limit on roads typically leads to a high percentage of accidents and fatalities each year in most developed countries. Unless the vehicle's speed can be limited or controlled, drivers are able to drive at dangerously high speeds, risk lives, and attract costly speeding fines after being photographed by a 'speed camera'. This may happen due to lack of awareness of the current speed limit in a particular area, or drivers not being careful enough to check their current vehicle speed. This paper describes a prototype system that can help reduce the over-speeding problem by fitting a transmitter on road signs and a receiver on vehicles. A maximum speed limit data signal is broadcasted continuously from a road sign, and a vehicle fitted with a wireless receiver module can detect the speed limit signal. The system can compare the vehicle's current speed with the speed limit and trigger an audible alarm (and alert message) to indicate that the speed limit has been exceeded. A working prototype has been designed, built and tested successfully using an 8-bit embedded microcontroller. Using appropriate transceiver modules, it is possible to implement Vehicle-to-Vehicle (V2V) and Vehicle-to-Infrastructure (V2I) communications in order to implement a wide range of different and useful functions, including collision detection, vehicle identification and even the processing of fines for traffic rule violations. This paper describes the design details of an over-speeding warning system and addresses several other practical applications of wireless technologies for improving road safety. It is hoped that this research will encourage further research into V2V and V2I applications by demonstrating how low-cost wireless communications modules and low-cost microcontrollers can be used to perform several different useful functions. If products like the devices described in this paper are marketed and sold, they could save lives and promote safer driving by simply annoying or constantly warning drivers who exceed the current speed limit, using a loud audible alarm which cannot be disabled by the driver, and warning of nearby dangers.
\end{abstract}

(C) 2017 The Author(s). Published by TAF Publishing.

\section{INTRODUCTION}

Exceeding the maximum speed limit on a road is usually among the top reasons for road accident fatalities. The 'Traffic Coordination General Directorate' at the Ministry of Interior (UAE) reports the following motor vehicle accident statistics for the year 2015 [1]:

- There were 675 deaths and 6863 injuries recorded on UAE Roads.

- Sudden swerving caused 919 accidents $(19.19 \%$ of all accidents), and 143 deaths (Number 1 cause of fatalities).
- Failure to maintain sufficient safe distance between vehicles caused 623 accidents (13.01\%), and 76 deaths.

- Misjudgment of road users, 556 accidents (11.61\%), and 75 deaths.

- Lack of attention, 499 accidents (10.42\%), and 73 deaths.

- Failure to comply with lane rules, 388 accidents (8.1\%), and 63 deaths.

- Entering a road without making sure it is clear, 349 accidents and 32 deaths.

- Over-speeding caused 281 accidents, and 82 deaths (Num-

\footnotetext{
Corresponding author: Samuel N. Cubero

†Email: scubero@pi.ac.ae
} 
ber 2 cause of road fatalities).

According to [2], 28 deaths out of a total of 255 road fatalities in Dubai (UAE) during 2009 - or about $11 \%$ of road deaths - were related to over-speeding and losing control of a motor vehicle. During 2009, a total of 112 people were also injured due to speeding-related accidents, 49 of which were severe or moderate injuries leaving permanent scarring or some form of permanent disability (e.g. inability to walk, paralysis, etc.). Although over-speeding is not among the leading causes of accidents, it is ranked as the 2 nd leading cause of death on roads during the year 2015 in the UAE due to the severity of collisions. Fatalities due to overspeeding is a serious problem that can be mitigated through various means such as:

1. Continuous education of teenagers and the general public regarding the serious consequences of over-speeding. 2. Traffic authorities could charge harsher penalties/fines for over-speeding (however, this will not stop those who are unaware of the maximum speed limit from over-speeding). 3. Speed limiters: These ensure that a vehicle cannot exceed a fixed maximum speed (say, $120 \mathrm{~km} /$ hour). The maximum speed setting cannot be adjusted by the driver. These devices are already fitted to many commercial trucks and taxis but most cannot automatically adjust the speed limit.

4. Speed limit controller (or warning device) may adjust the maximum speed limit based on the speed limit in a given traffic zone using GPS (Satellite navigation software), cell phone tracking or a DSRC (Dedicated Short-Range Communication) system attached to road infrastructure.

Unfortunately, the first two of these methods depend on human choice and can be rendered ineffective or circumvented by those who wish to violate traffic laws or those who are simply unaware of the current maximum speed limit in a given area.

Sometimes, drivers may fail to notice a maximum speed limit sign, perhaps due to distraction (not looking in the direction of the sign), or the speed limit sign may be difficult to see due to obstructions like signs, trees or other foliage. Sometimes, drivers leaving an $80 \mathrm{~km} /$ hour speed limit zone may turn into a new street or enter a new traffic zone with a $60 \mathrm{~km} /$ hour speed limit, without seeing the sign or without being given any warning of the new lower speed limit. Hence, the driver can be charged with a speeding fine due to lack of awareness of the speed limit in that area, especially if the driver is new to that area. It is important for the driver to be aware of the maximum speed limit in each area at all times.

\section{LITERATURE REVIEW}

Over the last two decades, extensive research has been conducted leading to the development of several different kinds of technologies in the area of wireless communications:

- 'Vehicle-to-Vehicle' (V2V) communication [2-5]

- 'Vehicle-to-Infrastructure' (V2I) communication [6-8]

- 'Vehicle-to-Vehicle-to-Infrastructure' (V2V2I) [9]

- Collision Warning Systems (CWS) [8]

The word 'Infrastructure' refers to stationary transceivers or wireless modules attached to a fixed location, such as a power pole, bridge, overpass, traffic light or a road sign. V2V and V2I communications are not yet ubiquitous, nor widely implemented, mainly because not all automobile manufacturers have included such computing and communication hardware as a 'standard feature' in modern vehicles. V2V2I is a 'hybrid' of V2V and V2I and exploits the advantages of both types of communication methods [9] by combining their features. Many highend luxury cars also feature a variety of different 'Collision Warning Systems' which can communicate with compatible vehicles (that can detect a CWS broadcast), and these devices can warn drivers of potential danger or imminent collision based on relative speed and proximity data from onboard sensors (such as distance sensors or machine vision image analysis). Most of the prototypes developed for the systems described above employ fairly expensive longrange data communications hardware, such as cell phones, 801.11 Wireless networks and even 'WiMax'. Such prototypes are quite complex and expensive to implement on a large scale.

GPS enabled speeding alarm systems are now quite popular and used by many different taxi companies, however, these units are quite expensive and do not come as a 'standard' feature on most new vehicles, except for many 'top end' luxury vehicles. GPS systems work quite well in most developed areas, however, they do not work in tunnels and they are not very reliable when driving under metal bridges or when there are many tall buildings surrounding a vehicle driving on narrow roads, such as in central business districts within a modern city.

A physical prototype of a very simple DSRC system was developed by Ahsan et al. [10] in 2014, however, this system only employed a wireless speed sensor and did not have the ability to warn or alert the driver of actual overspeeding because data communication was only 'one way' (V2I). Its main purpose was to automate the detection of 
an over-speeding vehicle (similar to the function of modern radar sensors or 'speed cameras') by transmitting speed information via a wireless (radio) link from the remote 'wheel' to a monitoring computer that displays 'speed' data from the wireless speed sensor. This system also had no way of automatically adapting to different or new speed limit settings in different traffic zones and cannot warn for over-speeding.

Jing describe a 'wireless' over-speeding detection system which reads RFID tags embedded in the road, using an onboard RFID tag reader [11]. [12] describe an automated over-speeding detection and reporting system which uses 'machine vision' technology (CCD cameras and software-based image analysis), however, this system uses expensive camera hardware and MATLAB software, and is still unable to provide the driver with a warning about overspeeding.

This paper demonstrates how very low cost electronic hardware and sensors can be implemented into existing vehicles and traffic infrastructure, as 'add-ons', for detecting an over-speeding vehicle, warning an over-speeding driver to slow down, and setting new 'speed limits' based on the data transmitted from a road sign so that the driver can be made fully aware of the new 'speed limit' in each new traffic zone that is entered. Devices like this provide audiovisual information to ensure the driver is aware of the current speed limit at all times. This study is significant because it demonstrates and describes several different kinds of novel practical applications that can enhance road safety, using very low cost wireless communications hardware.

\section{METHODOLOGY}

\section{A. Speed Limit Indicator and Over-Speeding Warning}

Many new vehicles are fitted with speed alarms but these require manual pre-setting by the driver each time the vehicle enters a new speed limit. The system described in this paper repeatedly sends the current speed limit from a road sign to a computer (mounted on a vehicle) via shortrange wireless technology. A display panel informs the driver of a speed limit change.

If the motorist exceeds the broadcasted speed limit, a warning buzzer will be triggered to alert the driver. This device can increase the awareness of motorists who are unintentionally speeding and encourage them to slow down. It may also help to reduce the number of accidents caused by over-speeding and reduce the economic burden caused by serious road accidents.

\section{B. Emergency Vehicle Warning System (EV)}

Emergency Vehicle drivers (Ambulance, Police, 'Fire and rescue' workers) often complain that other motorists are not aware of their presence and often do not 'give way' and make a clear path for them to proceed through congested traffic. The EV application will serve to warn drivers further ahead, in front of the EV. Current methods of sirens and flashing lights are only effective if people are close enough to hear or see them. Allowing emergency vehicles a less congested passage through traffic will reduce their travel times and improve road safety. This may help to save lives by reducing the likelihood of a collision with another vehicle, and allowing EV drivers to arrive at their destinations much faster.

\section{Motorcyclist Proximity Alert (MPA)}

Motorcyclists can die very easily in a road accident. Collisions between cars and motorcycles are quite common because most drivers cannot see motorcycles as easily as other bigger vehicles. The MPA system will alert drivers of motorcyclists in their 'blind spot'. This application is similar to the EV algorithm except it uses a different address number.

\section{Project Objectives}

The project goals are outlined below:

- Create a working prototype of a car to model a standard motor vehicle. This involves the design of a speed sensor for the car and a circuit to control motor speed.

- Design and then construct the onboard circuitry and wireless networking protocols to receive and display the speed limit. (This will be fitted to the model car).

- Create speed signs that repeatedly transmit the speed limit. This involves designing the speed sign and the connection of the transmitter beacon.

- Demonstrate wireless technologies in a variety of different practical applications. Applications include speed limit detection and triggering an over-speeding alarm to warn a driver, a Motorcycle Proximity Alert (MPA) application, and an Emergency Vehicle (EV) application - where the motorcycle or EV transmits a proximity warning signal.

- Perform and record a series of experiments to test a numb- 
er of parameters affecting the range of the project design and its applications. (Assessment of these parameters will lead us to determine the optimum parameter conditions for all designs - These will be incorporated into the existing designs).

- Design and create a device to limit and control the area, range and direction of the electromagnetic waves for the emergency vehicle application.

This paper describes the design, functions and different modes of operation for a prototype DSRC (Dedicated Short-Range Communication) system comprised of two main modules - each with built-in microcontrollers (executing embedded control software), namely: (1) The Vehicle module; and (2) the Infrastructure module, attached to a (stationary) road sign. The DSRC system described in this paper will be referred to as OSWA (Over Speeding Warning Alarm). A 'Remote Controlled' (RC) toy car is used as a 'test vehicle', fitted with the OSWA system (comprised of an 8-bit AVR microcontroller, wheel speed sensor, a wireless receiver module, a LED display screen, a motor speed controller and a power supply / battery). OSWA calculates the test vehicle's speed using an onboard speed sensor connected to one wheel. The 'Smart Road Sign' or SRS has a built-in microcontroller and transmitter that repeatedly broadcast the actual 'speed limit' for that traffic area. The 'speed limit' data are received and stored in OSWA and an alarm is activated (including a warning message) if the current vehicle speed exceeds the 'speed limit' sent by the SRS.

For the sake of brevity, details about antenna design, range testing, 'dead zones' (unreliable data reception), error checking, communication protocol design and microcontroller software algorithms were omitted, but can be supplied by the authors.

Each design with a transmitter module requires a different set of maximum range characteristics. These characteristics (for each of the transmitter designs) are outlined below:

- SRS Application-Optimizes range to minimize the chance of 'overlap' from nearby signals but attains a minimum range equating to the width of the road. The range must also be suitable for higher speed moving vehicles to retrieve information successfully (i.e. small ranges may not allow higher speed vehicles to retrieve the signal; this is also dependent on software, and the number of transmission cycles per second).

- MPA Application-Necessary to minimize the range to transmit a maximum radial distance of $7 \mathrm{~m}$.

- EV Application-Maximizing the range of the transmitted signal. The area covered by the electromagnetic signal however must be limited to a forward bias direction. This is why the design of a suitable device to limit the range of the area is required.

At this stage, the model vehicle has the ability to receive information regarding speed limits, and the proximity of motorcycle and emergency vehicles. Since the model vehicle ( $\mathrm{RC}$ car) is not able to attain car-like road speeds, the model vehicle speed has been calibrated to an effective speed range to mimic that of a regular car (with accurate speed control).

The following sections describe the OSWA and SRS designs, basic components, and communications principles used for establishing wireless data transfer between the 'infrastructure' and the 'vehicle' microcontroller modules.

\section{E. Electro-Magnetic Wave (EMW) Signal Transmission}

Communication between the transmitter and receiver modules is achieved through radio communication. The transmitter and receiver modules make use of a UART [13] (Universal Asynchronous Receive-Transmit) interface within the microcontroller. A UART is a simple 'serial communications port' for sending data 1 bit at a time in groups of a 'byte' of data (or 10 bits per character: 1 start bit, 8 data bits, and 1 stop bit). Information is encoded and sent through the TX line of the microcontroller (micro) where it is transmitted through an antenna. Receivers in the range of the transmitter area are able to pick up the encoded radio transmission from a transmitter, pass these data to the UART interface of the microcontroller, and the data are decoded back to their original form. This is the basic process for wireless communication between the microcontrollers.

A microcontroller sends data from its UART to a transmitter module, then the signals are sent to an antenna. An alternating voltage applied at the center of the antenna causes electron currents to flow up and down the antenna [14]. The result is that electrical charges develop at the ends of the antenna, with one end being positive and the other being negative, alternating with the frequency of the carrier wave. This process produces an electric field in the space surrounding the antenna. Since electrons are moving up and down the antenna, a magnetic field is also produced [15].

Both electric and magnetic fields are produced around the antenna and will exhibit sinusoidal variation at the oscillator frequency. The oscillator drives the antenna at Radio Frequency (RF) producing rapid variations. 
This causes the electric and magnetic fields to break free from the antenna, producing an EMW (Electro-Magnetic Wave).

Modulation is the term used for EMWs being transported through the air on what is referred to as a carrier wave. Figure 1 shows three types of waves. The first wave, Part (a), is not modulated by any external signal wave and thus a constant signal would be retrieved at the receiver end. A separate signal transmitted from an Amplitude Modulated (AM) transmitter will affect the carrier wave by altering its amplitude. This is shown in Part (b) of Fig. 1. The amplitude of the carrier wave is altered and highly sensitive to static interference, especially electrical noise.

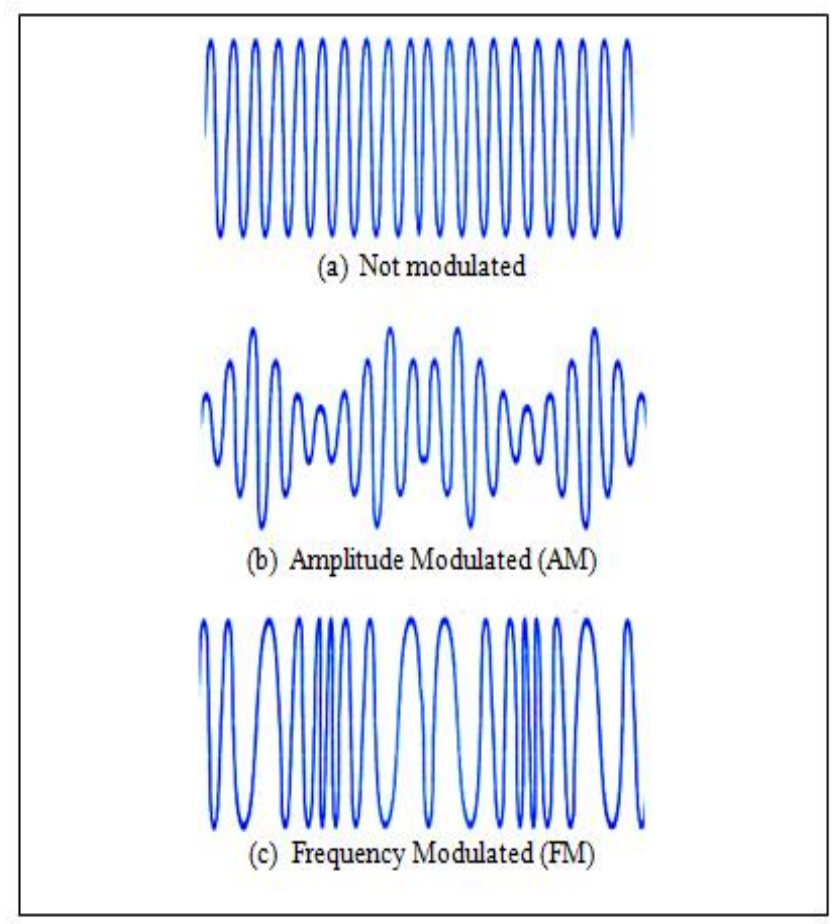

Fig. 1 . (a) Unmodulated signal; (b) AM signal; (c) FM signal

Frequency Modulation (FM) EMWs use a different approach to Amplitude Modulation (AM) EMWs. This form of modulation affects the frequency of the carrier wave rather than the amplitude. Part (c) of Fig. 1 shows an FM carrier wave with the frequency changing over time. Typically, AM transceiver (transmitter / receiver) packages require less power to transmit over larger distances and are less expensive than FM packages. However FM transceiver packages can handle transmissions at the extremes of the electromagnetic spectrum, unlike AM packages.

\section{F. Data Transmission between Microcontrollers}

There are several different ways to send and receive data between microcontrollers. Some common methods include UART, SPI, TCP/IP, and JTAG. Our project utilized Universal Asynchronous Receiver/Transmitter (UART) data transfer methods that were readily available onboard the AT90S8535 microcontroller. UART is a standard communications component that is provided by most microcontrollers to enable full-duplex serial communications.

The UART takes bytes of data and transmits the individual bits in a sequential manner, 1 bit at a time. At the destination, another UART reassembles the bits into complete bytes. The UART section of the Atmel AVR AT90S8535 microcontroller chip has two significant pins, 14 and 15 , where all the information is either transmitted or received.

AM EMW transmission systems are susceptible to electrical noise which may alter what the receiver retrieves. Methods were developed to counteract electrical noise and these are outlined in a later section.

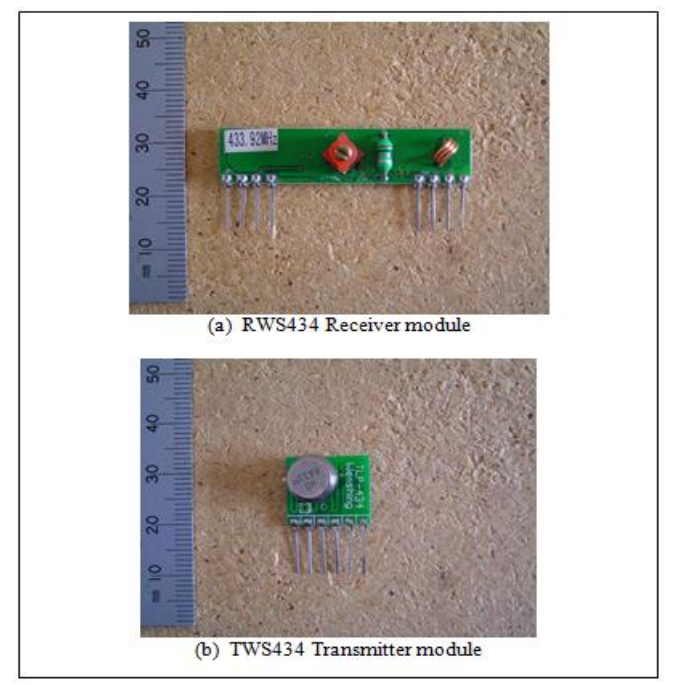

Fig. 2 . AM Wireless (a) Receiver; and (b) Transmitter modules

The UART also has limitations. Since this method of transferring data is undertaken in a sequential manner, only one bit at any time can be transferred through the interface; like a First-In-First-Out (FIFO) 'stack'. Data are transmitted only one bit at a time (serial communications), hence, the data transmission rate is limited. Introducing parallel lines to the system may improve this limitation; however regardless of how many parallel lines are used, there will always be a limitation to the data rate. 
The UART of the AT90S8535 microcontroller is, however, capable of transferring and receiving data fast enough for this project and its applications. Controlling the direction and the range of radio waves can be achieved by blocking a particular section of the transmission source. Certain materials have the ability to reflect the majority of EMWs with only a small percentage being absorbed and given off as heat. Aluminium is one element with this reflective ability. An alternative method is the use of reflective netting. Sizing the netting mesh to one-hundredth the wavelength of the carrier wave will allow the reflection of the majority of EMWs within the netting.

Figure 3 shows significant noise levels within the AM transmission. A 'decoupling circuit' built into the receiver module minimized the effect of noise and thus minimized the chance of triggering a logic low signal (resulting in corrupted data).

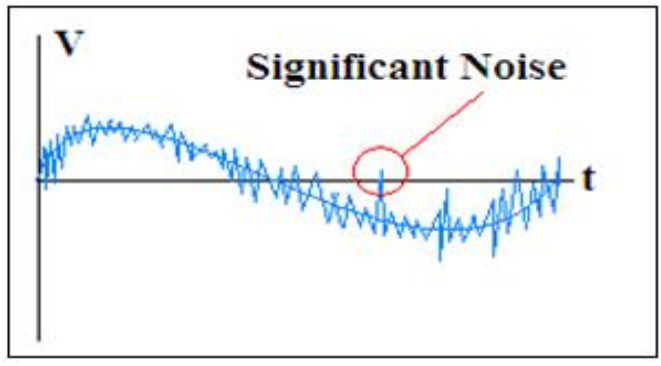

Fig. 3 . Example CRO readout for a varying digital output signal (AM)

The $433.92 \mathrm{MHz}$ AM transmitters and receivers were chosen over competing sets due to their low cost and simplicity of design. Being able to directly implement the package onto prototyping boards proved beneficial during general testing. This was also one of the reasons for choosing AM transmitters instead of FM transmitters. FM transmitters are also less common than AM transmitters and an FM transmitter usually requires greater power to deliver information at the same data rate as an AM transmitter.

FM transmitter signals do not suffer static (like in Fig. 3) and thus provide higher accuracy for data transmission than AM signals. This would eliminate the need for a decoupling circuit.

\section{G. Aerials}

Different aerial lengths were tested for RF range. Single core wire was used and a range of lengths was tested.
Recording the range achieved for the different aerial lengths showed the general relationship between the distance achieved and the length of the aerial. The cross-sectional area of the aerial affected the resistance of the wire and thus the range of transmission. The greater the cross-sectional area of the wire, the lower the resistance, and therefore, the greater the range. Even though the aerial cross-sectional area was not changed, the effect of altering the transmitter antenna resistance would have the same effect on range and thus was taken into account when interpreting the results from the range testing experiment.

Controlling the direction of radio waves required a significant amount of work. Attempting to propagate radio waves required a sophisticated aerial design. These aerial designs require an array of multiple aerials on top of each other. A 'base plate' creates a support for the radio waves to propagate off. Without a 'base plate', many of the radio waves will be wasted and the signal will not propagate as expected. The 'base plate' propagates the signal from each aerial producing the overall increase in signal strength for the main aerial design.

\section{H. Range Requirements}

Electromagnetic waves emitted from a transmitter have the ability to expand in a spherical shape as shown in Fig. 4. The strength of the transmitter will determine both the distance they travel and the strength of the signal over that distance.

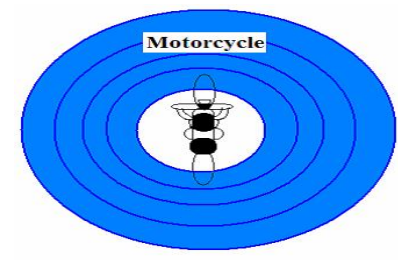

Fig. 4 Resulting transmission area of the motorcycle beacon (top view)

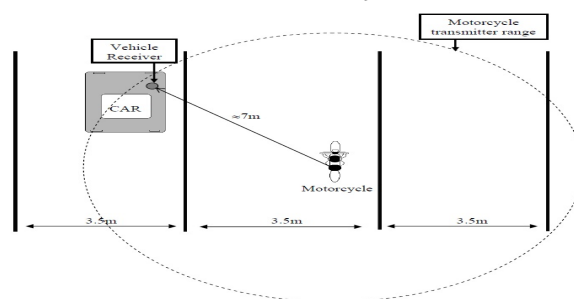

Fig. 5 . Limiting the range of the motorcycle beacon (for MPA system) 
It is important that the motorcycle proximity alert system is only activated when motorcycles are in close proximity to the motor vehicle to ensure that motorists do not become desensitized to the alarm. For this reason the motorcycle transmitter has been limited to a range of approximately $7 \mathrm{~m}$, as shown in Fig. 4, to provide adequate proximity warning.

The area that the emergency vehicle application covers was controlled much like a channel. This is shown in Fig. 6. The Emergency Vehicle (EV) transmitters have been designed to only alert vehicles in front or ahead of the EV vehicle. When the EV beacon is detected by these vehicles, internal alarms will activate to request motorists to give way or clear a path for the EV. For vehicles travelling on the road at $100 \mathrm{~km} /$ hour, a range distance of $60 \mathrm{~m}$ does not give much time for cars to get out of the emergency vehicle's path. In fact, within this distance, drivers of vehicles would most likely be able to hear the siren before the signal is received by the in-car receiver module, however, $60 \mathrm{~m}$ is sufficient for our experimental purposes.

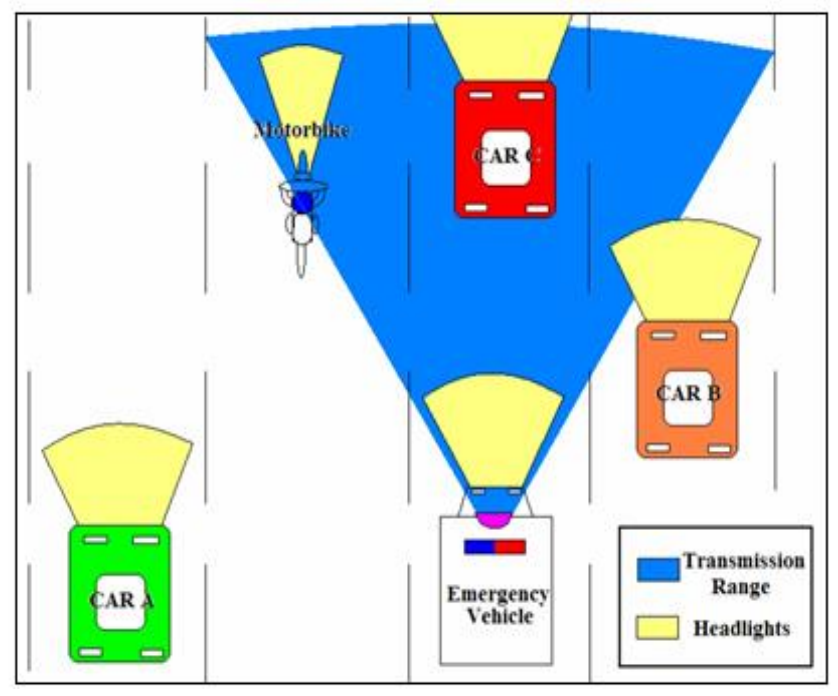

Fig. 6. Transmission area of the emergency vehicle (EV beacon)

More powerful transmitter/receiver modules and/or using propagation techniques would need to be used to significantly increase the transmission range for the EV beacon. The propagation of the signal would likely be the most useful. Using a 'whip antenna' that has a series of aerials set out inside the antenna, instead of using a single core wire, will help to achieve greater range.

Such designs are capable of magnifying the distance that a similar single-core non-grounded aerial covers by
4.5 times. When compared to the project model transmitter/receiver, a whip antenna installation would be capable of achieving distances in excess of $250 \mathrm{~m}$ (the theoretical maximum is $270 \mathrm{~m}$ ). This distance is sufficient for a 'real world' EV transmitter.

\section{Achieving the RF Direction Requirement}

Electromagnetic waves, like sound waves, have the ability to reflect, to be absorbed, and to diffract. A design to minimize the field of propagation requires certain materials to reflect and absorb radio waves. This will help stop EV signals from reaching other vehicles either behind or parallel to the emergency vehicle, so their alarms do not need to be triggered, as shown in Fig. 6 .

There are several methods of blocking electromagnetic waves. This project explored two such methods. These were wire-mesh 'netting', and reflection via material properties. Both these methods have the ability to block radio signals through reflection and/or absorption principles.

Aluminium foil has highly reflective properties with both electromagnetic (radio) and light waves. In this project, aluminium foil was used as it has greater accuracy than netting (also, creating a grid with small tolerances using limited manufacturing tools would be difficult). The outside casing would preferably be cylindrical since these directional radio transmitters are mounted on vehicles and will thus be exposed to significant wind forces. Using a cylindrical shape for the casing, with the front lip being of a spherical nature, would improve the aerodynamics of the design. Alternatively, placing the design inside the vehicle in front of the windshield would be equally effective. Radio waves have the ability to travel though glass and most of the signal would be able to pass through. This method of implementation would eliminate the need for an aerodynamic design and thus reduce the overall complexity of the design.

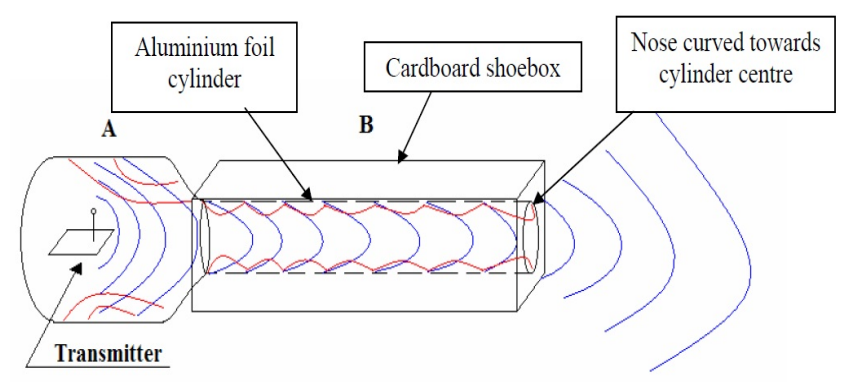

Fig. 7 . Controlling the direction of electromagnetic waves (EV beacon) 
Due to electromagnetic wave diffraction properties, a reduction in the aperture would lead to undesirable diffraction occurring. The design had to be completely rid of holes, particularly very small holes, as it is at these points where the greatest diffraction occurs with electromagnetic waves. Lining the inside of the pipe with several layers of aluminium foil helped achieve this and added to both the reflectivity and the concave nature inside the pipe.

Figure 7 shows the basic radio signal blocking design. Section $A$ of the design is a metal container from a spray gun and is made entirely of thin aluminium. Attachment $B$ is a long rectangular shoe-box which encased an aluminium foil cylinder to achieve the required length of the design. The blue waves indicate those which are being transmitted by the beacon. The red waves indicate those being successfully reflected from the design walls. It should be noted that Fig. 7 only shows the first phase of reflection in a model environment. Normally, the transmitted and reflected signals would result in a series of reflections occurring before the $\mathrm{RF}$ signals that reach the opening at the end of section $\mathrm{B}$. The interior of the path that the radio waves flow through was lined with several layers of aluminium foil to minimize the number of small holes through which the signal can diffract.

The signal that finally leaves the opening at section $B$ is now a controlled radio signal in reference to the volume covered. The volume covered by this design was in the form of a frustum (i.e. a truncated cone shape having a small angle of spread) and would be directed one way only. The distance that the waves travelled outside the end opening would depend on the strength of the signal. This is directly related to both the number of successful reflections within the design and the size of the antenna. As it was desirable to maximize distance for the emergency vehicle application, using the recommended antenna length of $35 \mathrm{~cm}$ was essential for optimum performance and the reflections within had to be limited. Each time a radio signal hit a reflective material, although the majority of the signal would be reflected, a portion of the signal would be absorbed and given off in the form of heat. If there are too many reflections, the signal strength of the transmitted data would leave the opening successfully but would not necessarily be strong enough to provide a satisfactory range.

\section{J. Free-for-all Networking}

The most common way of networking computers or microcontrollers is to have all transmissions linked back to a central hub or multiple central hubs that continually manage all transmissions, as shown in Fig. 8.

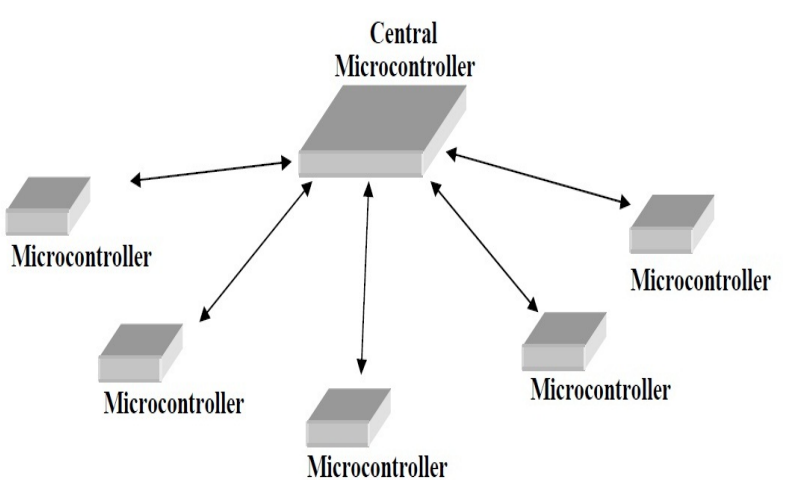

Fig. 8. Star network layout

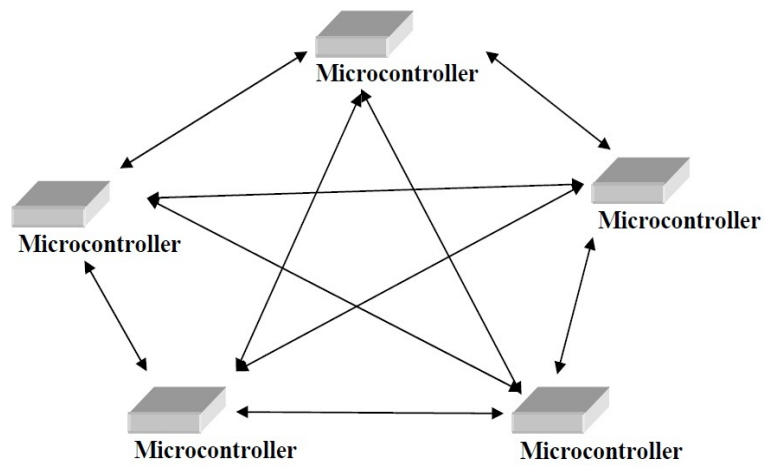

Fig. 9. Free-for-all communication network

A networking technique called 'free-for-all networking' can be used to effectively run multiple microcontrollers in a wireless network without a central master microcontroller devoted to managing the data transmissions of multiple slave microcontrollers. This networking method allows every microcontroller that is within wireless networking range to be its own master, and to talk to any other microcontroller directly without communicating through an intermediary or series of microcontrollers. This is shown in Fig. 9.

As with other networking methods, 'free-for-all networking' has advantages and disadvantages. For low cost wireless networking, the advantages of 'free-for-all networking' generally outweigh the disadvantages, thus, it is the preferred method for wirelessly interfacing multiple microcontrollers (if each unit can both transmit and receive).

Since the AM transmitters and receivers used in the design only had a frequency of $433.92 \mathrm{MHz}$, the limitation of the maximum reliable transmission speed was exper- 
imentally proven to be 2400 bps. Developing a suitable network to handle multiple microcontrollers that could enter or leave the network at any time was heavily reliant on the efficiency of the protocols (or communication rules). If the protocol required a large number of bytes to be sent back and forth for initialization then a large amount of radio transmission time would be wasted. 'Free-for-all' networking eliminates the need for initialization protocols as it simply uses a 'wake' byte. The 'wake' byte is usually followed by the address of the intended recipient microcontroller, which may be one or more bytes long, which is then followed by the data command byte(s). Transmitting data this way allows any microcontroller to talk directly with any other microcontroller within the network.

However, 'free-for-all networking' does have limitations. If there were hundreds of microcontrollers all trying to transmit their data every second or when the next transmission gap occurred after that second, then there would simply be too many signals being sent over the radio frequency for every microcontroller to be included. The likelihood of this situation occurring is dependent on the number of bytes being sent per transmission, on the gap detection time, and on how often each microcontroller needs to send a transmission. This could be overcome by either using a higher transmission frequency or multiple radio frequencies.

If 'free-for-all networking' was to be used for a small number of microcontrollers that were transmitting large amounts of data, then some microcontrollers may 'hog' the RF line due to the large number of bytes being sent sequentially in a single transmission. This would mean that the delays between transmissions from other microcontrollers would be larger than desirable, which would decrease efficiency and increase the delay time for other shorter and more urgent transmissions.

To eliminate this, transmissions can be broken up into smaller segments. So for example after three data command bytes are sent, it could wait for a time slightly longer than the gap detection time, thus allowing for any other microcontrollers to detect a gap and to start their own transmission. Meanwhile the original microcontroller should wait for the next transmission gap then send any remaining data command bytes.

The purpose of 'gap identification' is to detect when an asynchronous wireless serial communication transmission has stopped (radio silence) so that another micro will be able to begin a new transmission. Only one micro on the serial bus or network should 'talk' (or transmit) on the net- work at any time, and all the others must listen. The 'active micro' (or 'microcontrollers') must respond or act on such data. This is important to ensure that there are no 'data collisions' on the serial bus. It is imperative to prevent the condition where two or more bytes are being transmitted at any given time, because this will 'confuse' all the 'listeners' on the serial bus (or 'free-for-all' network).

Since this project did not require every microcontroller to have its own unique address, a generic 16-bit address was assigned to all microcontrollers. However, because the software design took addressing into consideration, there was no need to restructure the software. This meant that to create a number of microcontrollers that would operate in the same network, each microcontroller could easily be assigned a new 16-bit address number to provide uniqueness to individual microcontrollers or to different groups of microcontrollers.

The software was also designed to be able to easily 'daisy-chain' multiple bytes together. This allows the byte length of the addresses used to be easily adjusted. For example, if an 8-bit address was used then there would be 254 different combinations, since the number 255 is used as a 'wake' command and zero cannot be transmitted. Therefore there could be 254 different microcontrollers operating uniquely from each other that could come and go at any time within the same wireless network.

If a 16-bit address is used, then there would be 64,516 different microcontrollers that would be able to operate uniquely from each other and could come and go at any time. The number of unique addresses is given by (254)a where ' $a$ ' is the number of bytes 'daisy-chained' together. The same numerical principle could be applied to the number of unique data commands that were received by the microcontroller. K. Gap detection

Development of an effective networking system such as 'free-for-all networking' required all transmissions to be dependent on each other. This means that every microcontroller would continually check for periods of null transmission (no data). These null transmissions are commonly referred to as 'gaps', meaning that there is a gap between signals being sent on the radio frequency. By detecting the length of these transmission gaps, microcontrollers are able to wait for an appropriate time to send their information so that information isn't sent when another microcontroller is sending, as this would cause the information to be mixed and result in a garbage signal. Only 1 microcontroller should be transmitting at a time.

The timing of these gaps is based on the number of 
consecutive times the timer interrupt has been triggered without the value (that is received) changing. If there has been no change in 1728 timer interrupts, which is approximately 0.06 seconds, then a 'gap detected' flag is set. If the gap detection time was shorter than 0.06 seconds then the reliability would quickly decrease. Any gap detection time greater than 0.06 seconds is considered wasteful of time between transmissions.

The reliability was directly proportional to the timing of the gap, thus, the shorter the timing, the less time the micro had to measure to determine if a transmission was being sent or not and so it was more likely that the delay was too quick. By tuning the gap detection time, an efficient and reliable method of sorting data transmissions amongst multiple microcontrollers was developed.

Tuning the gap detection time was only possible by creating a program designed to flood the RF with data where only one bit of each byte was high, which thus produced the longest gap possible while data were being transmitted. For example if microcontroller ' $x$ ' attempted to transmit its own signal while the RF was being flooded by the gap detection program running on microcontroller ' $y$ ', then it would indicate that the gap detection time of microcontroller ' $\mathrm{x}$ ' was too short and needed to be increased. Using this method, it was possible to determine a gap detection time that would produce a $100 \%$ success rate for recognizing transmission gaps.

The gap detection was responsible for triggering two important functions, namely, the transmitting functions and the receiving functions. When the gaps were detected on the RF, this gave the micro an opportunity to transmit any information that was waiting to be sent. However, when there were no gaps detected (e.g. signals were being sent by another microcontroller), and the data are for that particular 'active' or 'awake' micro, then the receiver function was triggered to capture and process each byte being received by the receiver interrupt (stored in a 'buffer' memory; the size of which had to be large enough to avoid 'overflow' errors or lost data).

\section{OSWA System Components}

The hardware for the first vehicle-mounted OSWA (Over-Speeding Warning Alarm) is shown in Figs. 10 and 11 , including the motor speed control knob, an audible speaker (buzzer) and a LCD (Liquid Crystal Display) screen. These components were later mounted on a custom designed and manufactured double-sided PCB (Printed Cir- cuit Board) and housed in a protective case as shown in Fig. 10.

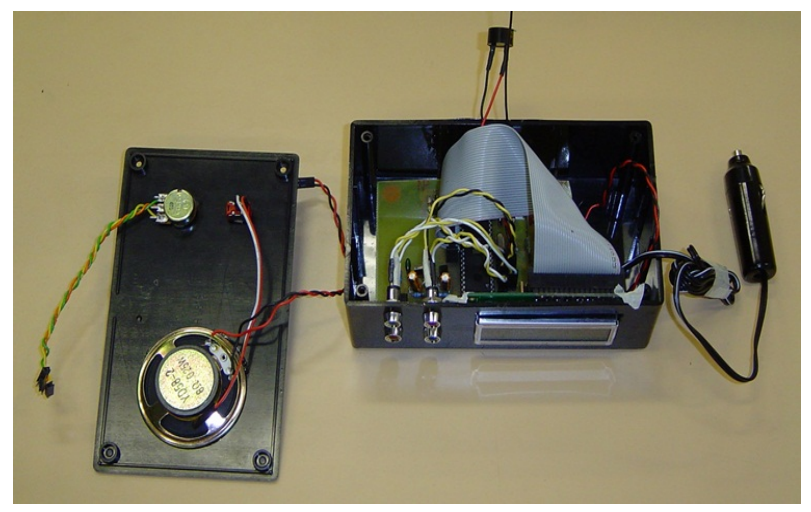

Fig. 10 . OSWA PCB prototype inside a protective case (for the vehicle)

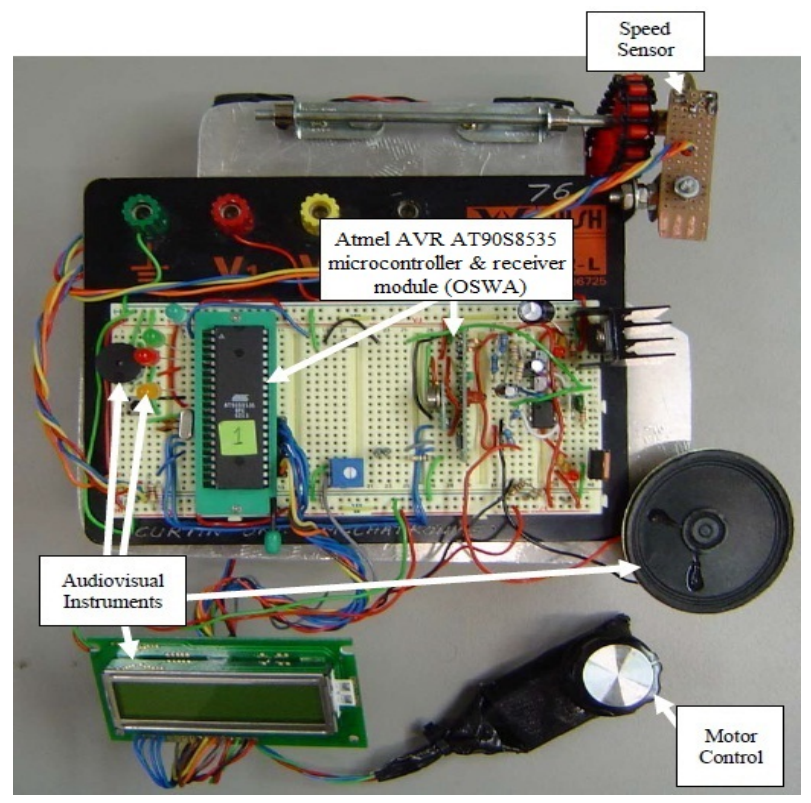

Fig. 11. Electronic components of the OSWA (on a prototyping board)

The PCB for the stationary transmitter module (SRS) that is mounted on the 'infrastructure' (e.g. road sign for new speed zone) is shown in Fig. 12 (shown without the protective case). The SRS includes the transmitter module (TWS434). Ideally, it should be placed at a very high position, at least several meters above the ground (perhaps at a 3 or 4 meter height above the ground, ideally on an overhead bridge or traffic light), to minimize interference and poor signal strength due to obstructions between the transmitter (on the road sign) and the receiver (the OSWA module on the vehicle). Other vehicles such as large trucks 
and other cars may weaken transmitted data signals because AM signals are very susceptible to interference from metal objects and obstructions. For example, it is possible for the metal body of a large semitrailer truck, or a large metal container, to corrupt or block the speed limit signal sent from an ordinary 'speed limit sign' to a motor vehicle with an OSWA, if the truck or metal body is taller than the OSWA vehicle. The transmitter should ideally be placed above all traffic in order to maximize the reliability of data reception.

The speed sensor is a non-contact through-beam IR (Infra-Red) optical sensor (H2A11 'slotted sensor') that is interrupted by the metal of a rotating metal disk attached to the driven wheel, the speed of which is controlled manually by the operator (using the 'Motor Control' knob, which is a simple potentiometer connected to an ADC 'Analogue to Digital Converter' input pin of the microcontroller). The rotating metal disk has holes drilled into it to allow light pulses to be detected by the optical sensor and such pulses are counted by the AVR AT90S8535 microcontroller, as shown in Fig. 13.

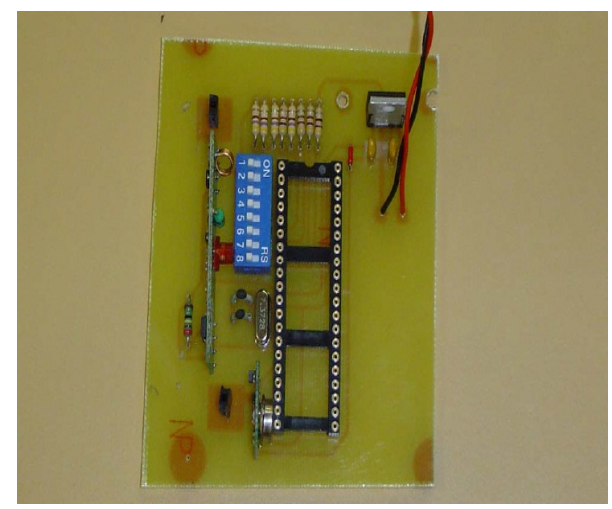

Fig. 12. Transmitter circuit mounted on 'infrastructure' (SRS or beacon unit)

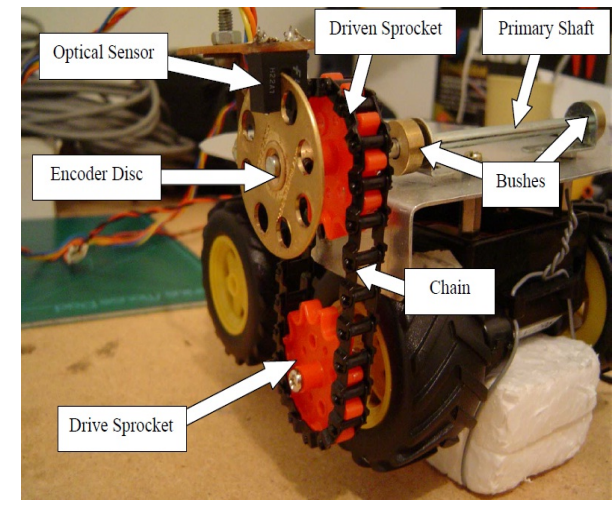

Fig. 13 . Radio controlled toy car with OSWA system

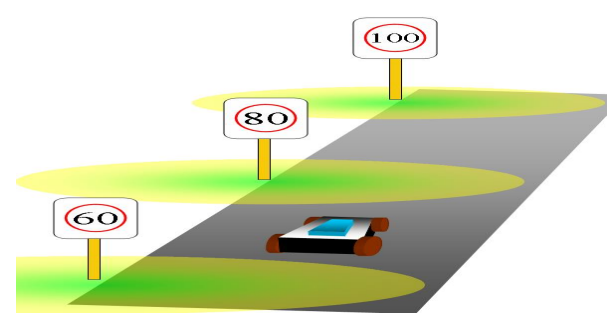

Fig. 14 . Each SRS (Smart Road Sign) broadcasts a speed limit (yellow zones)

\section{Setting a New Speed Limit}

The simple procedure described in this section explains how a simple I2V (Infrastructure to Vehicle) communication system works between a SRS (Smart Road Sign) and an OSWA system mounted on a vehicle. Since this is a very simple 'half-duplex' communication system, data is only sent 'one way', or from the SRS to the OSWA (because in this project, there was only one transmitter on the SRS, and one receiver - on the vehicle holding the OSWA). By fitting an additional transmitter module on the vehicle, and an additional receiver module on the SRS, full-duplex (or two-way) data communications can be implemented for all devices (both the OSWA and the SRS).

\section{Half-Duplex Wireless Communications}

The OSWA prototype system described in this paper only implements I2V (Infrastructure to Vehicle) communications. The transmitter module (beacon) is mounted on a road sign (a 'speed limit sign' - or SRS, 'Smart Road Sign'). For 'half-duplex' operation, the speed limit data value is being continually broadcasted by the SRS at 2400 bps. The OSWA (on the vehicle) is always monitoring its UDR (UART Data Register - or serial port), connected to the receiver module, checking for incoming data from an SRS beacon. Once the OSWA is within radio range of the SRS, it quickly adopts the new speed limit value and compares its current speed to this SRS data. If the current speed is above the broadcasted speed limit, an audible warning alarm is triggered and new speed limit information is displayed on the LCD screen. The OSWA maintains this same 'maximum speed limit' until it is changed again by a different SRS broadcasting a different speed limit.

2) Full-duplex wireless communications

True 'free-for-all' V2I (Vehicle to Infrastructure), I2V (Infrastructure to Vehicle) and V2V (Vehicle to Vehicle) network communications can be achieved if each microcon- 
troller is fitted with both a transmitter and a receiver module. There are many different ways to design a 'free-forall' networking protocol, each with their own advantages and disadvantages, however, great care must be taken to ensure that correct data are received by the correct microcontroller. There are two main types of network communications: (1) 'One to One' connections (with only one micro talking to another micro); and (2) 'One to Many' connections, where one microcontroller can send common data to two or more selected microcontrollers that need the same information.

For setting the maximum speed, a 'One to Many' connection method may be desired, however, it is easier to keep operating in 'half-duplex' mode most of the time, as described earlier, broadcasting a common speed limit value to all passing vehicles. If the SRS is fitted with a receiver, it can respond to and detect an incoming transmission from an OSWA that detects that its vehicle is actually over-speeding. The SRS can record information like the vehicle's identification details (e.g. registration plate number, driver details) and the excessive speed - which can be used to process a speeding fine.

Owners of OSWA devices also have the option to set the alarm to trigger at a set speed just below the 'speed limit' (say, 5 or $3 \mathrm{~km} /$ hour below the speed limit) so drivers can receive advanced warning and avoid going over the speed limit. For 'free-for-all' networking, the SRS could repeatedly send out 'wake-up' calls within its RF zone. This 'wake up' call is a simple transmission that is repeatedly sent out to invite OSWA systems within range to 'handshake' (establish a communication link) and begin transferring data. Initially, before initial contact, both SRS and OSWA systems are 'asleep', or not engaged in any data communication. When the OSWA receives a 'wake up' call from the SRS (Smart Road Sign), the SRS can proceed to transmit the new speed limit data, and any other crucial warning messages about nearby traffic dangers. This connection and communication process can occur completely automatically between one vehicle and another vehicle (fitted with a compatible OSWA, including motorcycles), and between vehicles and a nearby SRS.

\section{RESULTS}

To examine all the factors likely to have an impact on the RF transmissions, a series of tests were performed involving several different software and hardware factors. The software variables tested were error checking resolution, transmitter buffer size, and the number of transmission repeats per byte. The hardware factors tested were transmitter input and signal voltage, the length of the antenna on the receiver and on the transmitter, the transmitter antenna resistance, and the receiver filtering resistance.

These tests were important for determining the different transmission ranges that could be achieved for reliable data transmission. Once completed, such information allowed us to implement the primary project objectives. The motorcycle application in particular required these tests to be performed, since a $7 \mathrm{~m}$ radial range must not be exceeded so that misleading information is not received by vehicles in further lanes. (Ideally, the motorcycle RF range should be no less than $4 \mathrm{~m}$.).

TABLE 1

IMPACT OF BATCH SIZE ON ACCURACY

\begin{tabular}{lllll}
\hline \hline & Beacon & Car & Motorcycle & Emergency Vehicle \\
\hline Error checking resolution & 3 & 3 & 3 & 3 \\
Transmitter supply and signal voltage & 5 & 5 & 5 & 5 \\
Transmitter antenna resistance & 0 & 0 & 0 & 0 \\
Transmitter antenna size & $35 \mathrm{~cm}$ & $35 \mathrm{~cm}$ & $9 \mathrm{~cm}$ & $35 \mathrm{~cm}$ \\
Receiver antenna size & $1.5 \mathrm{M} \Omega$ & $1.5 \mathrm{M} \Omega$ & $1.5 \mathrm{M} \Omega$ & $1.5 \mathrm{M} \Omega$ \\
Transmitter buffer size & 19 & 19 & 19 & 19 \\
Number of repeats per byte & 5 & 5 & 5 & 5 \\
\hline \hline
\end{tabular}

Finally, the speed sign tests required a controlled transmission range, referred to as 'zones'. Both the project and its applications involved controlling the range to some extent, so these series of RF tests were intended to provide 
a sound understanding of both the advantages and disadvantages of the factors affecting the overall transmission range.

The OSWA (Over-Speeding Warning Alarm) system was built successfully to implement 'half-duplex' communications between a SRS (Smart Road Sign, with the transmitter) and an OSWA (with a receiver module). Demonstration videos showing this system working successfully under various different modes of operation are on YouTube at [16-20].

Wireless communication hardware for the SRS ('speed limit' beacon), OSWA (Car), MPA and EV applications were built and tested successfully. After much experimental testing, optimal operating parameters were found (see Table 1).

The receiver module (RWS434) has two outputs, digital out and linear out. Since the microcontroller's UART input is connected directly to the receiver module, the signal being sent to the UART must be a digital signal, thus the digital output is used instead of the linear output. However, leaving the linear output disconnected can cause problems with interference on the digital output signal. It was discovered that linking a large resistive load between the linear output pin of the receiver module and ground produced a more stable digital output signal. This was done to leak a small amount of power to the ground thus helping to eliminate possible standing waves.

Initially, during the software design, a $1 \mathrm{M} \Omega$ resistor was used to link this linear output pin to ground. This resistance value was discovered accidentally and seemed to filter out a large amount of interference with only a slight decrease in range. Yet this was simply an estimate and was later optimized using the results of many range testing experiments.

It was found that a receiver filtering resistor of 1.5 $\mathrm{M} \Omega$ provided the maximum range for reliable data transmission.

Selecting an antenna size of $9 \mathrm{~cm}$ for the motorcycle transmitter allowed the range of the transmitted signals to be restricted to a radius of $3 \mathrm{~m}$ (although, this could be increased to $7 \mathrm{~m}$ ). A $35 \mathrm{~cm}$ antenna was selected to give all other transmitters and all receivers a maximum range of approximately $60 \mathrm{~m}$. With the addition of a directional antenna the RF range of the signal was boosted substantially therefore allowing the emergency vehicle to have a much longer range. The filtering resistance for the RWS434 was selected as $1.5 \mathrm{M} \Omega$ because of the large increase in range (less noise) for such a small decrease in reliability.
Re-testing this value proved the reliability was definitely still within an acceptable level to meet the many situations that the project may encounter. The transmitter buffer size and number of repeats per byte were acceptable at 15 and 4 respectively. As this is the absolute minimum for the level of error checking resolution that was selected, it was decided that if one or two bytes was not received properly then error detection would not function properly and thus it would skip the transmission. To make sure that every transmission was detected the first time every time, the number of repeats was increased to five and therefore the transmitter buffer size also had to be increased to 19 .

For 'real world' motor vehicle applications, a $60 \mathrm{~m}$ radial $\mathrm{RF}$ range surrounding the SRS would be sufficient for establishing reliable communications between an OSWA (on a very fast moving vehicle) and a stationary SRS module (on a street sign). The faster a vehicle travels, the larger the $\mathrm{RF}$ range needs to be in order to avoid missing the detection of the SRS signal. Also, a much faster baud rate (wireless communication speed) would provide better overall transmission reliability and signal detection for faster moving vehicles.

\section{DISCUSSION}

Throughout the course of the project, there were several major problem areas encountered. Due to budgetary constraints, the availability of resources was limited. The project revolved around wireless networking between microcontrollers and this section was tackled first. In hindsight, the type of transmitter-receiver packages used could have been more thoroughly researched, possibly leading to an overall increase in the reliability of the range testing results. This would have saved many hours in the labs configuring the optimum parameters for the wireless modules, in particular the transmitter modules. Other wireless technologies may have also yielded better performance, such as 'ZigBee' (wireless modems, e.g. 'XBee' and 'XBee-PRO' transmitter/receiver modules), and IEEE 802.11, however, these wireless systems are considerably much more expensive than the basic AM transmitter and receiver modules used (shown in Fig. 2).

It was difficult to manipulate the configuration of the modules for testing purposes as they came pre-built. Limited knowledge of radio transmitters and receivers meant that we were not aware of decoupling principles and baseplate antenna design considerations. Pre-existing knowledge would have led us to design custom transmitter/re- 
ceivers allowing greater understanding for both the capabilities and limitations of generic transmitter-receiver modules through testing.

The design of a suitable algorithm to effectively enable communication using high AM frequencies between microcontrollers saw many unanticipated errors. Getting the modules to communicate to each other required a great amount of debugging time let alone further time spent optimizing the efficiency of the design.

Wireless technologies have a much higher degree of complexity than hard-wired applications. Even though the properties of electromagnetic waves are relatively simple, applying them in practice was more difficult. Electromagnetic waves are not visible to the naked eye so it was difficult to understand the extent of interference from various different sources. We did not initially appreciate that interference would affect the overall range and create areas of dead-zones (where no data could be received) to the extent realized.

Attempting the project a second time would see us move away from the transmitter-receiver packages altogether and move to Global Positioning Systems (GPS) that relate vehicle position to a database of speed limits. Since GPS is now being implemented in many newer vehicles, it could utilize an existing UART interface to communicate with automotive warning devices (e.g. GarminTM [21] sells a wide range of different GPS - or Global Positioning Satellite - sensors that can connect to a PC or any standard microcontroller UART and receive NMEA [22] data for determining location information from several satellites). Installing thousands of transmitter modules on individual speed signs would not be cost effective. However, this could be justified economically if SRS units are used for collecting and processing 'over-speeding' data, since they could be made and installed at much lower cost than dedicated speed camera boxes.

Also, low-cost speed cameras could be interfaced to a networked SRS computer. Integrating the MPA and EV alert features with already available GPS systems would be more economically viable, or GPS sensors could be connected directly to OSWA devices. Beacon transmitters mounted on motorcycles (for MPA) and emergency vehicles (EVs) may still be useful for providing proximity alerts to surrounding drivers who have a compatible receiver (similar to an OSWA). Although, such functions may also be implemented in a future downloadable 'road safety app' that can run on most popular mobile phone operating systems (like the iOS / iPhone, Windows Phone and Android).
The maximum range attainable by the current transmitter / receiver packages (in Fig. 2) was limited. Using antennas with well-designed base-plates would have significantly increased the range achieved by this transmitter / receiver package. Alternatively, choosing different wireless hardware with greater range capability could have extended the transmission range significantly (e.g. IEEE 802.11-type 'WiFi' transceivers, ZigBee, XBee, etc.).

The SRS devices described in this paper required each speed sign to have a power source for the transmitters. In rural areas, where there is no access to the grid, renewable energy alternatives should be considered (e.g. a small solar panel could recharge a small lithium battery to provide constant power).

The maximum speed of vehicles passing the speed signs is a limitation. This limiting factor is directly dependent on the radial $R F$ range. The radial range of the SRS transmitters will determine the maximum speed that a vehicle must not exceed in order to ensure successful data reception. The speed sign transmitter broadcasts the speed limit value about every half-second. On main roads and freeways, vehicle speeds are greater than those in suburban areas. The radial range therefore must be greater on these main roads and freeways to cater for higher speeds. A speed sign with a radial range of $30 \mathrm{~m}$, for example, will limit speeds to a maximum of $430 \mathrm{~km} /$ hour. Hence, this radial range is acceptable for this scenario.

To our best knowledge, the authors have not seen RWS434 and TWS434 wireless modules used in any other 'free-for-all' networking applications. Other researchers working on V2V and V2I research tend to use ZigBee, XBee and 802.11 technologies, which are more expensive alternatives. However, one robotics project that successfully employed RWS434 and TWS434 transmitters for 'half-duplex' communications was described by Aye et al. [23]. These researchers were able to achieve transmission distances up to 200 feet $(61 \mathrm{~m})$ - similar range results that were successfully tested by the authors, however, range is also dependent on antenna design. A wireless data link was used to transfer information between a ground-based controller and a microcontroller on a UAV (Unmanned Aerial Vehicle). Reliable manual flight control of the aircraft was achieved, however, only PWM (Pulse Width Modulated) signals were transmitted for manually controlling the speeds and positions of several different 'servo-motors' on the UAV. Therefore, there was no need for high precision data transfer, nor any stringent error checking of received bytes, since only 'rough positions' and 'approximate speeds' (at low data resolution) 
were required. Significant data errors could be tolerated with this type of application without seriously diminishing control performance.

Wireless communication between microcontrollers can be used as a basis for many future control and sensor applications. The applications need not be restricted to the automotive field. Applications linking 'intelligent' systems together to assist in automation are ideal derivatives of this project. Examples of 'intelligent' systems include home automation and security, communication between industrial robots and cooperative mobile robots, and relaying information around corners (multi-hop networking), e.g. communicating between different sectors of a mineshaft by passing information through a series of connected 'nodes' or wireless microcontrollers.

These kinds of applications are not yet implemented nor ubiquitous worldwide, nor are their features found in all modern commercially available vehicles. It is quite likely that these technologies will find their way into most modern motor vehicles and will become 'standard features' in perhaps 15 to 20 years from now.

\section{CONCLUSION AND FUTURE WORK}

All of the main objectives of this project were successfully completed and successfully demonstrated using low-cost AM transmitter and receiver modules. Wireless communication between microcontrollers was achieved providing the basic framework for the model motorcar. The Motorcycle Proximity Alert (MPA) and Emergency Vehicle (EV) applications were designed and tested. A total of three model vehicles were built. One model vehicle acted as the motorcar while the other two models simulated a motorcycle and an emergency vehicle. Speed signs were constructed to display and transmit the speed limit in a radial range. The range for the transmission was tested against a set of operating parameters. The maximum outdoor range of approximately $60 \mathrm{~m}$ was achieved with a $35 \mathrm{~cm}$ antenna size, a value of three for error checking resolution, antenna resistance of zero, filter resistance of $1.5 \mathrm{M} \Omega$, transmitter buffer size of 19 and the number of repeats per byte being five. All model vehicles were able to retrieve the speed limit from the speed signs and the MPA and EV proximity alert applications also worked as planned. The model motorcar had additional capabilities and was able to demonstrate the detection of the other two models under appropriate conditions through audiovisual aids (i.e. an audible alarm, warning lights and notices on the LCD screen).
This project is a major improvement on the work of [10] because it allows a new speed limit to be adopted by a speeding alert system onboard a vehicle, and it has the ability to detect nearby motorcycles and emergency vehicles fitted with a transmitter. It can be easily extended to allow full-duplex (bi-directional) data communications such as 'Vehicle to Vehicle' (V2V), 'Vehicle to Infrastructure' (V2I) and 'Infrastructure to Vehicle' (I2V) wireless communications - if the OSWA is fitted with an additional onboard 'transmitter'.

An OSWA may also broadcast a 'Please stop and help' request or an 'Emergency' call in the event of a problem, such as:

- A car hijacking (if a 'panic button' is pressed)

- Engine problems - e.g. radiator overheating / leakage, a flat battery or the engine just won't start.

- A flat tire (wheel) that needs changing; or

- An empty fuel tank

A special message can be displayed on the display screens of nearby OSWA units, requesting emergency assistance from passing motorists. A networked SRS would come in very handy in remote rural areas where mobile phone services do not work well (due to poor signal strength), or if the driver's mobile phone is flat and he/she forgot the battery charger and/or there is no other way to contact anyone for help. The OSWA may even be connected to airbag or crash detection sensors, so in the event of an accident or a collision, details of the accident and location information can be sent almost instantly to a nearby OSWA vehicle or a networked SRS. Such information can be sent immediately to emergency services and traffic authorities (including ambulance, police, vehicle towing and 'Fire \& Rescue' services). The OSWA computer could connect to and use an existing cell phone and its mobile network to contact emergency services.

One possible V2V application would be to warn the drivers of both vehicles about a potential collision if the relative speed between both vehicles is too high or unsafe given known 'safe' braking distances (similar to the 'collision alert' features in many modern 'high-end' luxury vehicles). Distance sensors (or range-finders) can be continuously monitored by a microcontroller in each vehicle. Distance to the nearest vehicle can be measured in all directions (forwards, backwards, left and right sides). This could significantly reduce road accidents caused by careless lane changes or sudden swerving caused by a driver who forgets to look quickly and check for a gap in an adjacent lane before changing lane. If the 'Smart Road Signs' 
(SRSs) are networked and made remote controllable, individual speed limits can even be adjusted or set immediately based on weather, traffic and road conditions. Also, drivers can be given advanced warnings to slow down or drive more carefully in that particular area if there happens to be dangerous traffic conditions ahead, such as a serious accident scene, poor visibility (due to fog or a sandstorm), slippery road conditions (like 'black ice', a chemical spill, or loose gravel) or perhaps an avalanche or a major roadblock ahead. The practical applications for wireless computers (with sensors) that can communicate data with each other are literally endless.

Since this project was very limited in scope, and none of the OSWA modules were fitted with transmitters (except for the MPA - Motorcycle Proximity Alert; and the EV - Emergency Vehicle applications, which behaved just like the SRS - 'Smart Road Sign' transmitters), a full-duplex 'free-forall' network was not implemented (containing many nodes, or many different microcontrollers), since only half-duplex (one-way) wireless communication was tested.

tab Hence, this paper only briefly discussed the possibility of implementing a true 'free-for-all' network. It is beyond the scope of this paper to describe in detail serial communications protocol design for a complete 'free-for-all' wireless network. This could be a topic for future research using the same or more reliable receiver / transmitter (or transceiver) modules, where each 'node' or micro in the network is capable of transmitting and receiving.

Future research will focus on how to exploit and harness the power of modern 'smart phones' for road safety applications because most of the essential hardware described in this paper are already 'built-in' features of modern mobile phones (e.g. GPS location sensors, cell phone networking, direct access to 'Emergency services' telephone numbers, and 'Bluetooth' connectivity to wireless speakers or hands-free headphones). It may even be possible for mobile phones to communicate with other nearby mobile phones even if they are not on the same cell phone 'network', just like they are 'nodes' in an 'ad-hoc' or 'freefor-all' network (i.e. Using automatic 'Wi-Fi' connectivity features). This will be a subject for future investigation.

\section{ACKNOWLEDGEMENTS}

The authors would like to thank the Department of Mechanical and Mechatronic Engineering, Curtin University (Perth, Western Australia) for the use of their mechatronic studio labs and equipment during the development of this project. Special thanks also goes to Jeff Pickles, Professor Kah-Seng Chung, John McLernon, Chris Sharpe, Joshua Portlock, Lee ISSN: $2414-4592$
Hearn, Anthony Foster and Kate Reynolds for all their help, advice and support.

\section{REFERENCES}

[1] Abu Dhabi News.Net, "32nd Gulf traffic week events to kick off on sunday," March 13, 2016. [Online]. Available: goo.gl/QoJqVX

[2] The Suraya Foundation, "Causes of accidents in Dubai 2009 (Dubai Police)-Statistical breakdown of accidents-by cause of accident 2009," 2009. [Online]. Available: goo.gl/Rj22RI

[3] S. Biswas, R. Tatchikou and F. Dion, "Vehicle-to- vehicle wireless communication protocols for enhancing highway traf-

fic safety," IEEE Communications Magazine, vol. 44, no. 1, pp. 74-82, 2006.

[4] Q. Xu, T. Mak, J. Ko and R. Sengupta, "Vehicle-to-vehicle safety messaging in DSRC," in Proceedings of the 1st ACM Inter- national Workshop on Vehicular ad hoc Networks, 2004, pp. 19-28.

[5] X. Yang, L. Liu, N. H. Vaidya and F. Zhao, "A vehicle-tovehicle communication protocol for cooperative collision warn- $\quad$ ing," in Proceeding 1st Annual International Conference on Mobile and Ubiquitous Systems: Networking and Services $\quad$ (MOBIQUITOUS), 2004, pp. 114-123.

[6] P. Belanovic, D. Valerio, A. Paier, T. Zemen, F. Ricciato and C. F. Mecklenbrauker, "On wireless links for vehicle-toinfrastructure communications," IEEE Transactions on Vehicular Technology, vol. 59, no. 1, pp. 269-282, 2009.

[7] J. Gosalvez, M. Sepulcre and R. Bauza, "IEEE 802.11p vehicle to infrastructure communications in urban environments," IEEE Communications Magazine, vol. 50, no. 5, pp. 176-183, 2012.

[8] S. Wang, Y. Cheng, C. Lin, W. Hong and T. He, "A vehicle collision warning system employing vehicle-toinfrastructure

communications," in Proceeding IEEE Wireless Communications and Networking Conference, 2008, pp. 3075-3080.

[9] J. Miller, "Vehicle-to-vehicle-to-infrastructure (V2V2I) intelligent transportation system architecture," in IEEE Intelli- gent Vehicles Symposium, 2008, pp. 715-720.

[10] M. Ahsan, J. McManis and M. S. J. Hashmi, "Prototype system development for wireless vehicle speed monitoring," $\quad$ in Proceeding 9th International Symposium on Communication Systems, Networks \& Digital Signal Processing (CSNDSP), 2014, pp. 287-292. 
[11] T. Jing, X. Li, W. Cheng, Y. Huo and X. Xing, "Speeding detection in RFID systems on roads," in Proceeding International Conference on Connected Vehicles and Expo (ICCVE), 2013, pp. 953-954.

[12] S. Malik, M. Iqbal, Z. Hassan, T. Tauqeer, R. Hafiz and U. Nasir, "Automated over speeding detection and reporting sys-

tem," in 16th International Power Electronics and Motion Control Conference and Exposition (PEMC), 2014, pp. 11041109.

[13] F. Dura, "The UART: What it is and how it works," January 13, 1996. [Online]. Available: goo.gl/91rsGz

[14] Yerkes Summer Institute, "The other side of crystal radio-Basics of AM radio transmission," 2002. [Online]. Available: goo.gl/g0JNkr

[15] "Energy in an electromagnetic wave," [Online]. Available: goo.gl/djc4rt

[16] "OSWA-Emergency vehicle detection," [Online Video file]. Available: goo.gl/EQE4Wb

[17] “OSWA-Motorcycle detection," [Online Video file]. Available: goo.gl/PIkr9i

[18] "OSWA-Speed alert front view," [Online Video file]. Available: goo.gl/rGTdnE

[19] "OSWA-Speed alert side view," [Online Video file]. Available: goo.gl/wKlUIH

[20] "OSWA-Varying speed limits," [Online Video file]. Available: goo.gl/kdeZPd

[21] Garmin, "Garmin GPS sensors," 2016. [Online]. Available: goo.gl/uGQK0F

[22] Wikipedia, "NMEA 0183," 2016. [Online]. Available: goo.gl/iP4Xq2

[23] T. S. Aye, P. T. Tun, Z. M. Naing, and Y. M. Myint, "Development of unmanned aerial vehicle manual control system,"

World Academy of Science, Engineering and Technology, vol. 42, no. pp. 392-396, 2008.

— This article does not have any appendix. — 